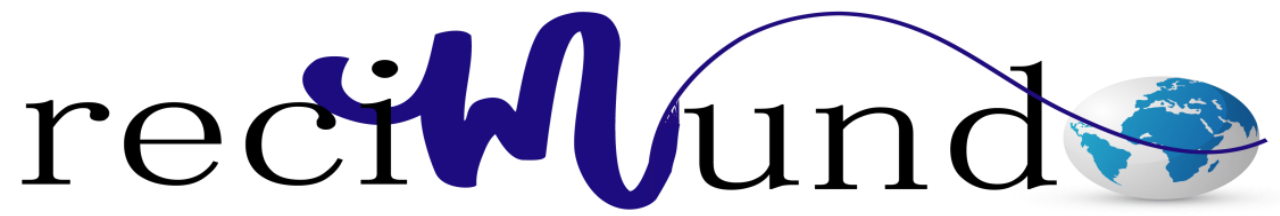

Revista Científica Mundo de la Investigación y el Conocimiento

Flerida María Alcívar Cedeño a ; Janina Alexandra Montalvan Espinoza ${ }^{\text {b; }}$ Marlin Manuel Alvarado Zuñiga ${ }^{c}$

Diseño de un modelo de costeo idóneo para la producción de quesos en microempresas comunitarias Salinas de Bolívar

Revista Científica Mundo de la Investigación y el Conocimiento. Vol. 2 núm., 1, febrero, ISSN: 2588-073X, 2018, pp. 413-426

DOI: $\underline{10.26820 / \text { recimundo/2.1.2018.413-426 }}$

Editorial Saberes del Conocimiento

Recibido: 05/12/2017

Aceptado: 15/02/2018
a. Universidad de Guayaquil; flerida.alcivarced@ug.edu.ec
b. Universidad de Guayaquil; janina.montalvanes@ug.educ.ec
c. Universidad de Guayaquil; marlin.alvaradoz@ug.edu.ec 


\section{Diseño de un modelo de costeo idóneo para la producción de quesos en microempresas comunitarias Salinas de Bolívar}

Vol. 2, núm. 1., (2018)

Flerida María Alcívar Cedeño; Janina Alexandra Montalvan Espinoza; Marlin Manuel Alvarado Zuñiga

\section{RESUMEN}

El modelo de costeo para producción de quesos ha ayudado a las empresas en responder al mercado con productos de mejor calidad a precios más competitivos. El control de costos como herramienta para que las organizaciones obtengan productos con una mayor eficiencia y eficacia. Analizando la rentabilidad del producto, el método del $\mathrm{ABC}$ ha contribuido eficazmente con el proceso de toma de decisión. Con el ABC, las microempresas pueden mejorar su eficacia y reducir costos sin sacrificar valor para el cliente. Esto también permite a las microempresas comunitarias, calcular el impacto de la reducción de costos y confirmar posteriormente, los ahorros logrados; el Costeo Basado en Actividades (ABC) es un método dinámico para la mejora continua de los procesos. Con éste método la entidad puede tener su particular ventaja competitiva basada en costos y así puede agregar valor continuo a sus clientes. La implantación del modelo de gestión basado en la metodología $\mathrm{ABC} / \mathrm{ABM}$, no solo le permite a la entidad conocer el costo de sus productos y servicios financieros, sino que va a determinar los centros de costos, y sobre los cuales ejercerá un control de costos a todo nivel de la organización. Con la aplicación del modelo en Excel que se plantea le permitirá mejorar los procedimientos, conocer

que actividades le generan valor al proceso y qué recursos optimizar, adicionalmente, va a considerar una integración y cohesión entre los niveles de la dirección, y el personal de negocios donde se generan los resultados. De la investigación realizada se presenta un modelo conceptual y práctico del procedimiento que lleva su implantación, seguimiento y evaluación del modelo dentro del proceso; este modelo se puede aplicar en los diferentes productos de las microempresas una vez definidos los recursos, las actividades, los direccionadores y el objeto del costo. Además se propone con la implantación del modelo contar con la información necesaria como soporte para la toma de decisiones.

Palabras clave: Modelo de costeo, elementos de costo, pymes, presupuestos, rentabilidad. 


\title{
Diseño de un modelo de costeo idóneo para la producción de quesos en microempresas comunitarias Salinas de Bolívar
}

Vol. 2, núm. 1., (2018)

Flerida María Alcívar Cedeño; Janina Alexandra Montalvan Espinoza; Marlin Manuel Alvarado Zuñiga

\begin{abstract}
The costing model for cheese production has helped companies respond to the market with better quality products at more competitive prices. Cost control as a tool for organizations to obtain products with greater efficiency and effectiveness. By analyzing product profitability and customer profitability, the $\mathrm{ABC}$ method has effectively contributed to the decision-making process. With $\mathrm{ABC}$, microenterprises can improve efficiency and reduce costs without sacrificing customer value. This also allows community micro-enterprises to calculate the impact of cost reduction and subsequently confirm the savings achieved; Activity Based Costing (ABC) is a dynamic method for continuous improvement of processes. With this method the entity can have its particular competitive advantage based on costs, so it can add continuous value to its clients. The implementation of the management model based on the ABC / ABM methodology, not only allows the entity to know the cost of its products, but will determine the cost centers, and on which will exercise a cost control at all levels of the organization. With the application of the model in Excel that raises will allow you to improve the procedures, to know which activities generate value to the process, what resources to optimize, will consider an integration and cohesion between the levels of management, and the business personnel where generate the results. The research carried out presents a conceptual and practical model of the procedure that leads to its implementation, monitoring and evaluation of the model within the process; this model can be applied to the different products of the micro once the resources, activities, indicators and the object of the cost have been defined. In addition, it is proposed with the implementation of the model to have the necessary information as support for decision making.
\end{abstract}

Keywords: Costs model, cost elements, smes, budgets, profitability. 


\section{Diseño de un modelo de costeo idóneo para la producción de quesos en microempresas comunitarias Salinas de Bolívar}

Vol. 2, núm. 1., (2018)

Flerida María Alcívar Cedeño; Janina Alexandra Montalvan Espinoza; Marlin Manuel Alvarado Zuñiga

\section{Introducción.}

La investigación está orientada a diseñar un modelo de costeo idóneo que permita mejorar los niveles de competitividad de las microempresas productoras de queso objeto de estudio, mediante el manejo eficiente de costos, consecuentemente el establecimiento de precios razonables, lo cual permitirá indirectamente incrementar ventas y mejorar la productividad. Pues del diagnóstico realizado se evidencio de manera preliminar que el proceso de costeo que disponen actualmente es empírico; consecuentemente ineficiente, que no les permita valorar en términos reales los costos en los que han incurrido en la producción, lo que a su vez impide conocer sus costos reales y por ende su margen de utilidad real, de manera que les permita tomar decisiones eficientes, eficaces y oportunas, que beneficien a la comunidad. (Aguilar, Ramírez, \& Barrón, 2007) (Peña, 2005)

Para realizar esta investigación se empleará el enfoque cuantitativo, porque se quiere evaluar la afectación de los insumos en los costos de producción, que actualmente carece de herramientas de control necesarias. El tipo de investigación será descriptiva y explicativa por que se empezará describiendo el problema investigado y luego se estudiará la causa efecto del mismo, que posibilite proponer una alternativa de solución. El método que se usará será el deductivo, estadístico, analítico y el diseño de la investigación será de tipo documental y de campo por que se revisara información secundaria y también se recogerá información de la fuente del problema, para lo cual se utilizará técnica de encuesta y el instrumento será el Cuestionario, el mismo que se lo realizara a los microempresarios de la comuna y una muestra 


\section{Diseño de un modelo de costeo idóneo para la producción de quesos en microempresas comunitarias Salinas de Bolívar}

Vol. 2, núm. 1., (2018)

Flerida María Alcívar Cedeño; Janina Alexandra Montalvan Espinoza; Marlin Manuel Alvarado Zuñiga

representativa de los habitantes del sector Salinas de Bolívar. (Ramírez Urquidy, Texis Flores, \& Aguilar Barceló, 2014) (Pérez \& Stumpo, 2002)

La justificación práctica de esta investigación radica en aspectos como: la evaluación de la necesidad de tener un modelo de costeo idóneo; así como el diseño del mismo, que se ajuste a las necesidades y conveniencias de los comuneros de quesos de Salinas de Bolívar. La justificación metodológica será la conceptualización y teorización de las variables inmersas o involucradas en el tema objeto de la investigación.

El objetivo de la presente investigación es diseñar un modelo de costeo $\mathrm{ABC}$ dirigido al sector micro empresarial comunitario cooperativa de fabricación de Quesos en Salinas de Bolívar.

\section{Metodología.}

La investigación está orientada a diseñar un modelo de costeo idóneo que permita mejorar los niveles de competitividad de las microempresas productoras de queso objeto de estudio, mediante el manejo eficiente de costos, consecuentemente el establecimiento de precios razonables, lo cual permitirá indirectamente incrementar ventas y mejorar la productividad. Pues del diagnóstico realizado se evidenció de manera preliminar que el proceso de costeo que disponen actualmente es empírico; consecuentemente ineficiente, que no les permita valorar en términos reales los costos en los que han incurrido en la producción, esto impide conocer sus costos reales y por ende su margen de utilidad real, de manera que ayude a tomar decisiones 


\section{Diseño de un modelo de costeo idóneo para la producción de quesos en microempresas comunitarias Salinas de Bolívar}

Vol. 2, núm. 1., (2018)

Flerida María Alcívar Cedeño; Janina Alexandra Montalvan Espinoza; Marlin Manuel Alvarado Zuñiga

eficientes, eficaces y oportunas, que beneficien a la comunidad. (Hernández, Fernández, \& Baptista, 2010)

\section{Propuesta.}

\section{Modelo de Costeo ABC}

Proceso de Asignación de Costos en el Sistema de Costos ABC

El Proceso de asignación de Costos en el Sistema ABC se ejecuta en tres etapas

a) Asignación de los costos a las actividades

- Localización de los Costos Indirectos en los centros

- Identificación y clasificación de las actividades

- Distribución de los costos del centro entre actividades

- Elección de los generadores del costo (cost-drivers)

- Reclasificación o reagrupación de las actividades

- Calculo del costo unitario de los generadores del costo

b) Asignación de los costos de las actividades y de los costos directos a los productos

- Asignación de los costos de las actividades a los materiales y a los productos

- Asignación de los costos directos a los productos

c) Determinación del Costo

- Costo final unitario

- Análisis e interpretación de los Resultados 


\section{Diseño de un modelo de costeo idóneo para la producción de quesos en microempresas comunitarias Salinas de Bolívar \\ Vol. 2, núm. 1., (2018)}

Flerida María Alcívar Cedeño; Janina Alexandra Montalvan Espinoza; Marlin Manuel Alvarado

\section{Localización de los Costos Indirectos en los centros}

Las microempresas comunitarias de Salinas de Bolívar se encuentran dividida en cuatro

Departamentos, que son:

Tabla 1.- Departamentos y costos indirectos en los centros.

\begin{tabular}{lccc}
\hline \multirow{2}{*}{ Departamento } & \multicolumn{3}{c}{ Elementos del Costo } \\
\cline { 2 - 4 } & Mano de Obra & CIF & Total \\
\hline Dirección & 1500 & 160 & 1660 \\
Administración & 980 & 70 & 1050 \\
Producción & 1300 & 1200 & 2500 \\
Ventas & 960 & 700 & 1660 \\
Total & 4740 & 2130 & 6870 \\
\hline
\end{tabular}

Nota: Alcívar (2017)

\section{Identificación y clasificación de las actividades}

La clasificación e identificación de las actividades es el resultado de las encuestas efectuadas al personal administrativo; y esto permitió determinar el costo que le corresponde a cada una de estas.

Tabla 2.- Identificación y clasificación de las actividades

\begin{tabular}{ll}
\hline Departamento & Actividades \\
\hline Dirección & Compra de materia prima, Transporte de insumos \\
\hline
\end{tabular}


Diseño de un modelo de costeo idóneo para la producción de quesos en microempresas comunitarias Salinas de Bolívar

Vol. 2, núm. 1., (2018)

Flerida María Alcívar Cedeño; Janina Alexandra Montalvan Espinoza; Marlin Manuel Alvarado Zuñiga

\begin{tabular}{ll}
\hline Administración & Contabilidad, Pago de personal y de proveedores \\
Producción & Análisis de la leche, Tratamiento, fabricación del Queso y Empacado \\
Ventas & Distribución, Facturación y Cobranza, \\
\hline
\end{tabular}

Nota: Alcívar (2017).

Distribución de los costos del centro entre actividades

La distribución de los costos de cada departamento se efectúa correspondiendo al costo de los recursos con las actividades, las que se determinaron de acuerdo a la información de las encuestas.

Tabla 3.- Distribución de los costos del centro entre actividades

\begin{tabular}{lcccc}
\hline \multirow{2}{*}{ Actividades } & \multicolumn{4}{c}{ Elementos del Costo } \\
\cline { 2 - 5 } & Dirección & Administración & Producción & Ventas \\
\hline Compra de materia prima & 1170 & & & \\
Transporte de insumos & 90 & & & \\
Contabilidad & & 350 & & \\
Pago a proveedores & & 200 & 200 & \\
Pago de personal & 460 & 700 & \\
Análisis de la leche & & & 1500 & \\
Tratamiento & & & & 700 \\
Fabricación del Queso & & & & 600 \\
Distribución & & & & 1900 \\
Facturación & & & & \\
Cobranza & 1260 & & & \\
Total & & & & \\
\hline
\end{tabular}

Nota: Alcívar (2017).

Elección de los generadores del costo (cost-drivers) 


\section{Diseño de un modelo de costeo idóneo para la producción de quesos en microempresas comunitarias Salinas de Bolívar \\ Vol. 2, núm. 1., (2018)}

Flerida María Alcívar Cedeño; Janina Alexandra Montalvan Espinoza; Marlin Manuel Alvarado Zuñiga

La elección de los generadores es importante para este modelo de Costeo ABC ya que estos generadores relacionan a las actividades con las demandas de los productos. Los generadores tomados son fáciles de medir, tomando en cuenta que son los que mejor representan la relación causa-efecto entre el consumo del recurso y la actividad del producto.

Tabla 4.- Cost Drivers

\begin{tabular}{llc}
\hline \multicolumn{1}{c}{ Actividades } & \multicolumn{1}{c}{ Cost Drivers } & Total \\
\hline Compra de materia prima & Numero de compras & 1700 \\
Transporte de insumos & Kilómetros recorridos & 90 \\
Contabilidad & Horas de trabajo & 350 \\
Pago a proveedores & Número de facturas pagadas & 200 \\
Pago de personal & Horas de trabajo & 460 \\
Análisis de la leche & Horas de trabajo & 200 \\
Tratamiento & Horas de trabajo & 700 \\
Fabricación del Queso & Horas de trabajo & 1500 \\
Distribución & Kilómetros recorridos & 600 \\
Facturación & Número de ordenes & 700 \\
Cobranza & vendidas & 600 \\
TOTAL & Volumen de producción & $\mathbf{6 6 7 0}$ \\
\hline
\end{tabular}

Nota: Alcívar (2017).

\section{Reclasificación de las actividades}

En esta parte del proceso se tomó en consideración que en los diferentes departamentos de la fábrica, existen actividades que se asemejan o son idénticas entre sí; a las que fueron 


\section{Diseño de un modelo de costeo idóneo para la producción de quesos en microempresas comunitarias Salinas de Bolívar}

Vol. 2, núm. 1., (2018)

Flerida María Alcívar Cedeño; Janina Alexandra Montalvan Espinoza; Marlin Manuel Alvarado Zuñiga

agrupadas para facilitar el proceso asignado en esta investigación. También se consideró la similitud de la finalidad y el mismo generador de costo.

\begin{tabular}{llc}
\multicolumn{2}{c}{ Tabla 5. Reclasificación de las actividades } \\
\hline \multicolumn{1}{c}{ Actividades } & \multicolumn{1}{c}{ Cost Drivers } & Total \\
\hline Distribución & Kilómetros recorridos & 600 \\
Transporte de insumos & Kilómetros recorridos & 90 \\
Compra de materia prima & Numero de compras & 1700 \\
Contabilidad & Horas de trabajo & 350 \\
Pago de personal & Horas de trabajo & 460 \\
Análisis de la leche & Horas de trabajo & 200 \\
Tratamiento & Horas de trabajo & 700 \\
Fabricación del Queso & Horas de trabajo & 1500 \\
Pago a proveedores & Número de facturas pagadas & 200 \\
Facturación & Número de ordenes & 700 \\
Cobranza & vendidas & 600 \\
Total & Volumen de producción & 6670 \\
\hline
\end{tabular}

Nota: Alcívar (2017).

\section{Cálculo del costo unitario de los generadores del costo}

El costo unitario del generador se obtuvo dividiendo los costos totales de cada actividad, para el número de generadores. Este costo unitario representa la medida del consumo de los recursos que cada generador crea en la actividad. 


\section{Diseño de un modelo de costeo idóneo para la producción de quesos en microempresas comunitarias Salinas de Bolívar \\ Vol. 2, núm. 1., (2018)}

Flerida María Alcívar Cedeño; Janina Alexandra Montalvan Espinoza; Marlin Manuel Alvarado Zuñiga

\section{Tabla 6.- Costo unitario de los generadores}

\begin{tabular}{llccc}
\hline Actividad & Generador & Costo & $\begin{array}{l}\text { Nro de } \\
\text { generadores }\end{array}$ & Costo unitario \\
\hline $\begin{array}{l}\text { Distribución } \\
\text { Transporte de insumos }\end{array}$ & $\begin{array}{l}\text { Kilómetros } \\
\text { recorridos }\end{array}$ & 690 & 40 & 17,25 \\
$\begin{array}{l}\text { Compra de materia } \\
\text { prima }\end{array}$ & Numero de compras & 2400 & 80 & 30 \\
$\begin{array}{l}\text { Facturación } \\
\text { Contabilidad }\end{array}$ & Horas de trabajo & 350 & 40 & 8,75 \\
Pago de personal & Horas de trabajo & 460 & 40 & 11,5 \\
Análisis de la leche & Horas de trabajo & 200 & 20 & 10 \\
Tratamiento & Horas de trabajo & 700 & 40 & 17,5 \\
Fabricación del Queso & $\begin{array}{l}\text { Horas de trabajo } \\
\text { Número de facturas }\end{array}$ & 1500 & 40 & 37,5 \\
Pago a proveedores & $\begin{array}{l}\text { pagadas } \\
\text { Volumen de } \\
\text { pobranza }\end{array}$ & 600 & 10 & 20 \\
Total & & 6670 & 12 & 50 \\
\hline
\end{tabular}

Nota: Alcívar (2017)

\section{Asignación de los costos de las actividades al producto}

Para asignar los costos de las actividades a los materiales y al producto, se basó en el número de los generadores que cada producto consumió o demandó de cada actividad, esto quiere decir que es el número de prestaciones con las que la actividad contribuyó en la formación del producto. 
Diseño de un modelo de costeo idóneo para la producción de quesos en microempresas comunitarias Salinas de Bolívar

Vol. 2, núm. 1., (2018)

Flerida María Alcívar Cedeño; Janina Alexandra Montalvan Espinoza; Marlin Manuel Alvarado Zuñiga

Las actividades que se realizan en el Departamento de Distribución y que se originan por las Ventas no repercute en el producto fabricado por lo que no es considerado en esta asignación de costos, ya que estos están direccionados en las actividades de los productos vendidos.

Tabla 6.- Costos de las actividades al producto.

\begin{tabular}{|c|c|c|c|c|}
\hline Actividad & Generador & $\begin{array}{l}\text { Costo } \\
\text { Unitario }\end{array}$ & $\begin{array}{l}\text { Nro de } \\
\text { generadores }\end{array}$ & $\begin{array}{l}\text { Costo } \\
\text { Total }\end{array}$ \\
\hline $\begin{array}{l}\text { Distribución } \\
\text { Transporte de insumos }\end{array}$ & Kilómetros recorridos & 17,25 & 40 & 690 \\
\hline $\begin{array}{l}\text { Compra de materia } \\
\text { prima } \\
\text { Facturación }\end{array}$ & Numero de compras & 30 & 80 & 2400 \\
\hline Contabilidad & Horas de trabajo & 8,75 & 40 & 350 \\
\hline Pago de personal & Horas de trabajo & 11,5 & 40 & 460 \\
\hline Análisis de la leche & Horas de trabajo & 10 & 20 & 200 \\
\hline Tratamiento & Horas de trabajo & 17,5 & 40 & 700 \\
\hline Fabricación del Queso & Horas de trabajo & 37,5 & 40 & 1500 \\
\hline $\begin{array}{l}\text { Distribución } \\
\text { Transporte de insumos }\end{array}$ & Kilómetros recorridos & 20 & 10 & 200 \\
\hline $\begin{array}{l}\text { Compra de materia } \\
\text { prima } \\
\text { Facturación }\end{array}$ & Numero de compras & 17,25 & 12 & 690 \\
\hline Contabilidad & Horas de trabajo & 30 & 40 & 2400 \\
\hline
\end{tabular}

Nota: Alcívar (2017) 


\section{Diseño de un modelo de costeo idóneo para la producción de quesos en microempresas comunitarias Salinas de Bolívar \\ Vol. 2, núm. 1., (2018)}

Flerida María Alcívar Cedeño; Janina Alexandra Montalvan Espinoza; Marlin Manuel Alvarado Zuñiga

\section{Asignación de los costos directos a los productos}

Después de haber asignado los costos directos del producto se procedió a sumar los costos directos, más los costos indirectos de fabricación, obteniendo los costos finales de producción.

Tabla 7.- Costos totales

\begin{tabular}{lccc}
\hline \multicolumn{1}{c}{ Actividades } & Cantidad & Costo Unitario & Costo Total \\
\hline Costos directos & 1350 & 30 & 40500 \\
Costos Indirectos & & 0,44 & 594 \\
Costos totales de fabricación & & 41094 \\
\hline
\end{tabular}

Nota: Alcívar (2017)

\section{Costo final unitario}

Para determinar con exactitud lo que cuesta producir el producto se considera el costo de fabricación, las facturas de venta y la recaudación, esto dividido para el costo unitario; de esta manera se obtuvo el costo final sobre las unidades vendidas

\section{Análisis e interpretación de los Resultados}

El precio de venta de cada queso en USD 3; precio para competir en el mercado, este precio comparado con el que se genera como costos unitario evidencia una diferencia a favor de la fábrica en USD 0,22 ctv., lo que equivale al $11 \%$ de utilidad neta una vez que se desglosaron todos los gastos de materia prima, equipo de trabajo con sueldos establecidos por ley y 


\section{Diseño de un modelo de costeo idóneo para la producción de quesos en microempresas comunitarias Salinas de Bolívar}

Vol. 2, núm. 1., (2018)

Flerida María Alcívar Cedeño; Janina Alexandra Montalvan Espinoza; Marlin Manuel Alvarado Zuñiga

reposición - mantenimiento de maquinaria, lo que motiva a los propietarios a estar satisfechos y continuar, mejorar, optimizar y agrandar con visión empresarial.

\section{Conclusiones.}

Las microempresas comunitarias de Salinas de Bolívar., no cuentan con un sistema de costos para los productos que ofrece, por lo que es primordial que las instituciones cuenten con la metodología de un sistema de costeo $\mathrm{ABC}$ que les permitirá contar con la información necesaria para mejorar la toma de decisiones.

Las microempresas al no contar con el Sistema de costeo ABC, pierde la oportunidad el costo real de los productos que presta a los socios clientes, desconoce el costo de cada actividad y el costo de cada Departamento, para conocer cuáles son más rentables.

\section{Bibliografía.}

Aguilar, J., Ramírez, N., \& Barrón, K. (2007). Conformación de la microempresa marginada en la frontera norte de México. Estudios Fronterizos, 8(15), 51-71.

Hernández, R., Fernández, C., \& Baptista, P. (2010). Metodología de la Investigación (5 ed.). México: McGraw Hill.

Peña, A. (2005). El sistema de información contable en las pequeñas y medianas empresas. Un estudio evaluativo en el área metropolitana de Mérida, Venezuela. Actualidad Contable Faces, 8(11), 67-79.

Pérez, W., \& Stumpo, G. (2002). Las pequeñas y medianas empresas industriales en América Latin. México: CEPAL.

Ramírez Urquidy, M., Texis Flores, M., \& Aguilar Barceló, J. (2014). El papel del capital humano y el aprendizaje en las microempresas de base social en Baja California. Estudios fronterizos, 15(29), 207-245. 